\title{
Creating an Anti-Racism Steering Committee
}

By Milton Justinsuthakaran and Mehr Karim, Barts and The London, QMUL, London, UK

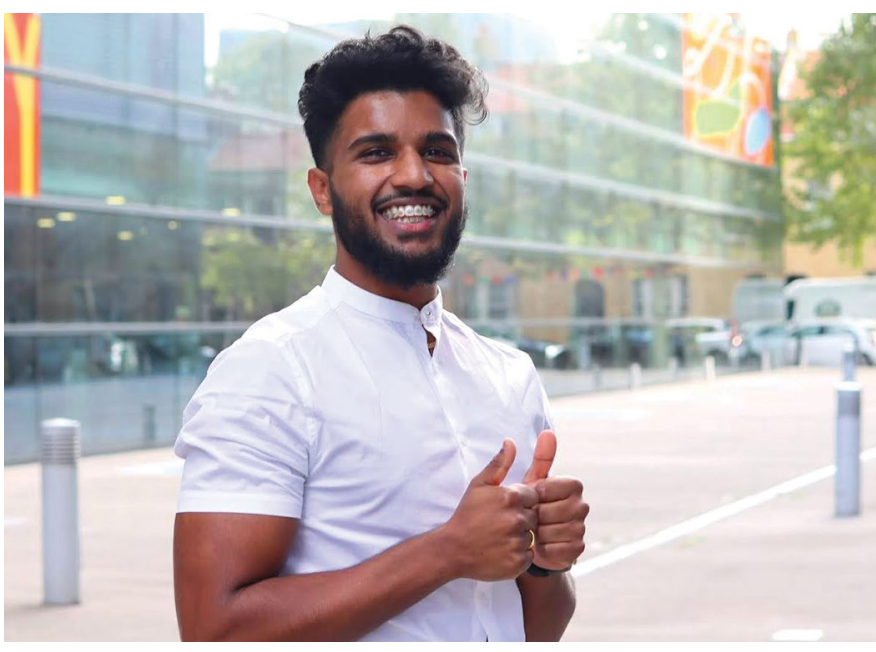

Milton Justinsuthakaran

$\mathrm{I}$ $\mathrm{n}$ light of the Black Lives Matter protests that surrounded us in the global zeitgeist, students at Barts and The London, Queen Mary University of London's (QMUL's) School of Medicine \& Dentistry, have come forward to discuss issues of racism and racial bias they have noted within their own experiences during their time at university. This conversation has branched out to the other departments within the School, now involving dentistry, medicine, physician associates, global health, pharmacology and neuroscience, leading to the formation of our Anti-Racism Steering Committee (ARSC). Our aim is to act as a formalised, long-term structure through which anti-racism approaches can be embedded into every aspect of the university experience for both staff and students. We have found the pan-departmental approach to be really useful, particularly because it allows us to collectively look into Faculty-wide issues and ensure that these conversations are not siloed or held in bubbles of good practice, but are shared with everyone to make sure we are all on the same page. We are in the process of sharing and implementing similar structures across all QMUL faculties.

Our governance structure reflects the multitude of departments involved through sub-groups, which is the case for dentistry. This dental sub-group was led by Student President, Milton Justinsuthakaran, as well as the ARSC's Dental Student Lead, Mehr Karim. Although there are commonalities between dentistry and other departments with issues of racism, there are individual pockets that need to be addressed within the degree itself which these sub-groups aim to rectify. We have started to identify issues through an anti-racism dental student survey aimed at all years and alumni. This survey brought forward both quantitative and qualitative data which we were able to

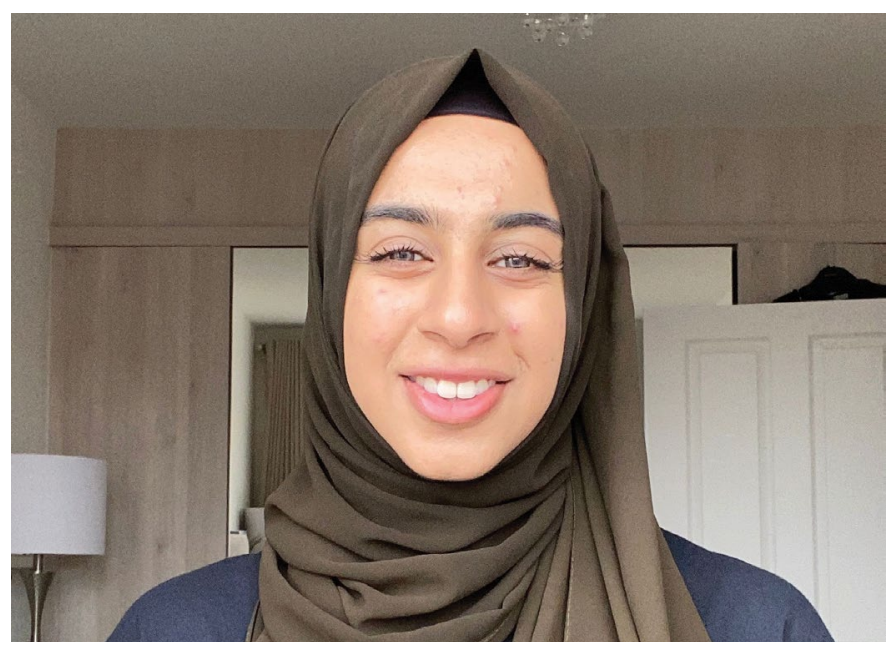

Mehr Karim

analyse and process into a summative report, allowing us to identify concerns within the dental school. The quantitative data highlighted patterns within student experience of racism while the qualitative data allowed us to identify key themes; gave the students a voice; and crucially allowed us to develop a deeper understanding of their concerns. It is worth noting that the inclusion of alumni experiences provided a valuable insight into life after dental school, shedding light on the challenges faced by alumni and the preparedness, or lack thereof, for race-related challenges following university.

A number of key themes transpired during the analysis of our results, unifying students across all year groups. It quickly became clear that students were concerned not only about their own experiences of racism and racial bias, but the effects on their peers and society as a whole. A predominant trend was the push towards a more diverse curriculum which draws examples from BAME (Black,

\section{'Our aim is to act as a formalised, long-term structure through which anti-racism approaches can be embedded into the university experience'} Asian and Minority Ethnic) sources when teaching. It is clear that
diseases manifest differently within various ethnic groups and these
differences are not highlighted in our studies to a sufficient degree.
One of our main goals at the Institute of Dentistry is to decolonise
the curriculum and this was reassuringly reflected in the student
opinion, with an overwhelming $79 \%$ of respondents feeling as
though the dental school could do more to address racial bias within
the curriculum. The committee aims to reform modules in which
we are predominantly taught about clinical signs with regards 
4 to lighter skin tones, as well as simultaneously addressing the unconscious biases we may have. Alongside this theoretical teaching, there was a strong desire for students to be practically equipped with the skills to tackle situations surrounding racism and racial bias should they arise. Only $40 \%$ of survey respondents felt comfortable in addressing incidents of racial discrimination if they witnessed them - a concern shared by alumni who noted a lack of preparedness when dealing with these issues in the workplace. In addition to teaching, students drew attention to the social environment of dental school, calling out issues such as microaggressions and divisions between students. A worrying 31\% felt as though their ethnicity prevented them from participating in BLSA (Barts and The London Student Association) extracurricular activities. As such, it was evident that a holistic and multi-faceted approach would be needed in order to tackle the wide range of concerns identified through our research.

In response to our findings, the Dental ARSC sub-group formulated six recommendations which were set out to tackle the full scope of student concern. We met with the Dean of Dentistry to inform him of our progress and discuss our next steps, and were met with his interest and support. Following a letter of his approval, we began working alongside a team of staff to begin implementing the changes necessary to create progress within the dental school. During this process, the dental sub-group have been updating, and will continue to update, the rest of the ARSC on the progress we make and the concerns we may have.

With regards to the COVID-19 pandemic, it has highlighted the inequalities in our BAME groups. Disadvantaged social and economic circumstances may have potentially contributed to the disproportionate healthcare impact on these ethnic groups. Sixty percent of Black residents and $50 \%$ of Bangladeshi residents in England and Wales live in London. At Barts, the patient population

\section{'There was a strong desire for students to be practically equipped with the skills to tackle situations surrounding racism and racial bias'}

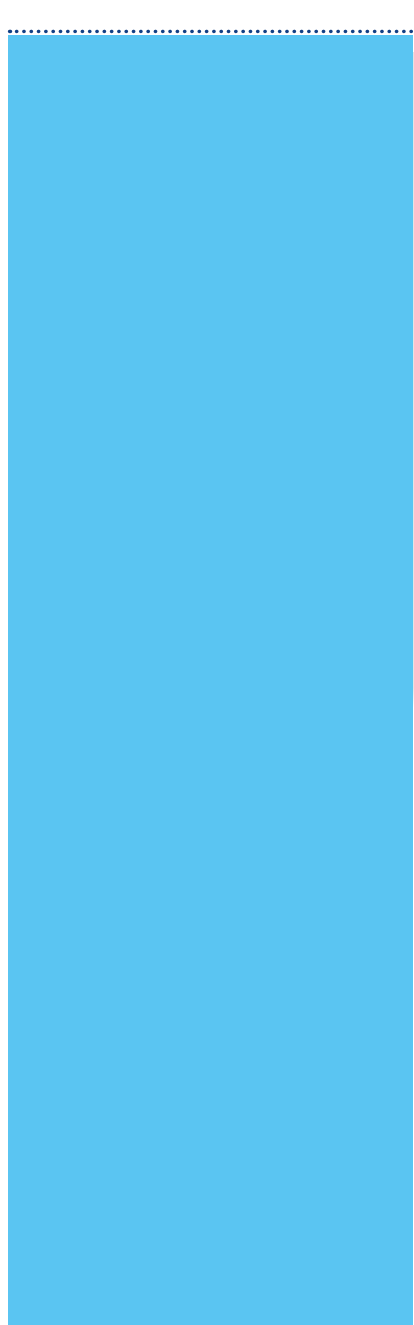

at the dental school and outreach centres is very diverse and we must be adequately trained in order to provide the best level of care. For us to become better dentists, there needs to be more ethnic representation of taught material in our curriculum to reflect today's multicultural society.

It is with all this mind that the ARSC was born at Barts and The London. However, to assume the issues mentioned are exclusive to this institution would be naivety, and hence this article was written to share the journey embarked upon. The aim is to encourage other institutions to also take the difficult step of introspection and elicit change to make our dentals schools go from good to great. 